\title{
Adhesión discal de la articulación temporomandibular: Revisión de la literatura
} Intraarticular adhesion of the temporomandibular joint: A review

Aránguiz $M^{*}$, Arriagada $C^{*}$, Gübelin $M^{*}$, Bornhardt $T^{* *}$, Iturriaga $V^{* *}$

\section{RESUMEN}

Los trastornos temporomandibulares son un conjunto amplio de patologías dentro de las cuales están las adherencias discales. Estas son una fijación permanente del disco articular a una de las superficies, debido a un aumento de la carga estática articular y posterior colapso del espacio articular superior o inferior, siendo provocado por una unión fibrótica entre estas superficies. Es necesario aumentar el reconocimiento de esta patología para su diagnóstico y tratamiento oportuno, por lo que el objetivo de este artículo es realizar una revisión en cuanto a la epidemiología, etiopatogenia, diagnóstico y tratamiento de la adhesión discal de la articulación temporomandibular.

Palabras clave: Articulación temporomandibular, adhesión discal, artrocentesis, artroscopia, trastornos temporomandibulares.

\section{SUMMARY}

Temporomandibular joint disorders are a wide range of conditions where there are the intraarticular adhesions of the temporomandibular joint. These are a permanent attachment to the articular disk surface due to an increase of static load and collapse articular joint space above or below, being caused by a fibrotic joint between these surfaces. It's necesary increase the recognition of this disease for an appropiate diagnosis and treatment, so the aim of this paper is to review regarding the epidemiology, pathogenesis, diagnosis and treatment of the discal adherence of the temporomandibular joint.

Key words: Temporomandibular joint, intraarticular adhesions, temporomandibular joint disorders, arthroscopy.

Fecha de recepción: 26 de mayo de 2014.

Aceptado para publicación: 3 de junio de 2014.

* Estudiante de Odontología. Facultad Odontología. Universidad de La Frontera. Temuco, Chile.

** Magíster y especialista en Trastornos Temporomandibulares y Dolor Orofacial, Departamento Integral del Adulto. Facultad de Odontología. Universidad de La Frontera. Temuco, Chile.

Aránguiz M, Arriagada C, Gübelin M, Bornhardt T, Iturriaga V. Adhesión discal de la articulación temporomandibular: Revisión de la literatura. Av. Odontoestomatol 2015; 31 (1): 19-23.

\section{INTRODUCCIÓN}

Los trastornos temporomandibulares (TTM) son un conjunto de patologías relacionadas a la articulación temporomandibular (ATM) y sus estructuras asociadas (1) los cuales pueden ser de diversa naturaleza y origen multifactorial (2,3). El 50\% de la población sufre algún signo o síntoma de TTM y aproximadamente un $7 \%$ se encuentra afectado por un trastorno bucomaxilofacial causante de dolor 
facial y mandibular (4). Dentro de los TTM se encuentran las adherencias y adhesiones del disco articular, los cual corresponden a un TTM de origen articular dado por la incompatibilidad de las superficies anatómicas y estructurales. Las adherencias discales son uniones esporádicas del disco a alguna de las superficies articulares, lo cual se libera durante la reanudación del movimiento articular (1); al contrario, las adhesiones discales (AD) son una fijación permanente del disco articular a una de las superficies articulares, debido a un aumento de la carga estática en la ATM y posterior colapso del espacio articular superior o inferior (5), provocado por una unión fibrótica entre estas superficies (1).

\section{EPIDEMIOLOGÍA}

Las adhesiones discales en la ATM ocurren con mayor frecuencia en el espacio articular superior (6) y se pueden dar a lo largo de toda superficie articular del hueso temporal, con el disco desplazado/luxado o en correcta posición. En un estudio publicado de un total de 450 pacientes examinados a través de resonancia magnética, 4 casos fueron diagnosticados como adhesiones discales, siendo 2 unilaterales y 2 bilaterales; lo que determina una frecuencia de $0,89 \%$ (3), no encontrándose reportes epidemiológicos en Chile.

\section{ETIOPATOGENIA}

La $A D$ es un fenómeno que puede explicarse mediante dos procesos:

1. El ácido hialurónico secretado por los sinoviocitos es el responsable de lubricar la articulación y así facilitar el movimiento articular, por lo que en primera instancia una inflamación en la sinovial o una sobrecarga mantenida de la articulación podría ocasionar fenómenos de hipoxia-reperfusión provocando la ruptura de las cadenas de alto peso molecular de este polisacárido mediado por fosfolipasa A2, con una secuencia de deposición de una cantidad de fibrina sobre los componentes articulares lo que podría evolucionar en la formación de tejido fibroso provocando la adhesión discal $(2,3,7)$ (Fig. 1).
2. La presencia de hemartrosis secundaria a macrotraumatismo o una intervención quirúrgica provocaría un proceso de reorganización de las fibras colágenas, neoformación capilar y transición a tejido cicatrizal promoviendo la fibrosis y provocando la adhesión discal $(2,3,7)$.

\section{DIAGNÓSTICO}

El diagnóstico de la adhesión discal de la ATM comienza con la anamnesis y examen clínico, los cuales se pueden complementar con técnicas imagenológicas para confirmar la patología $(8,9)$. La historia de traumatismos en la región mandibular (5) o de una intervención quirúrgica (19) son datos importantes de rescatar, al igual que parafunciones como

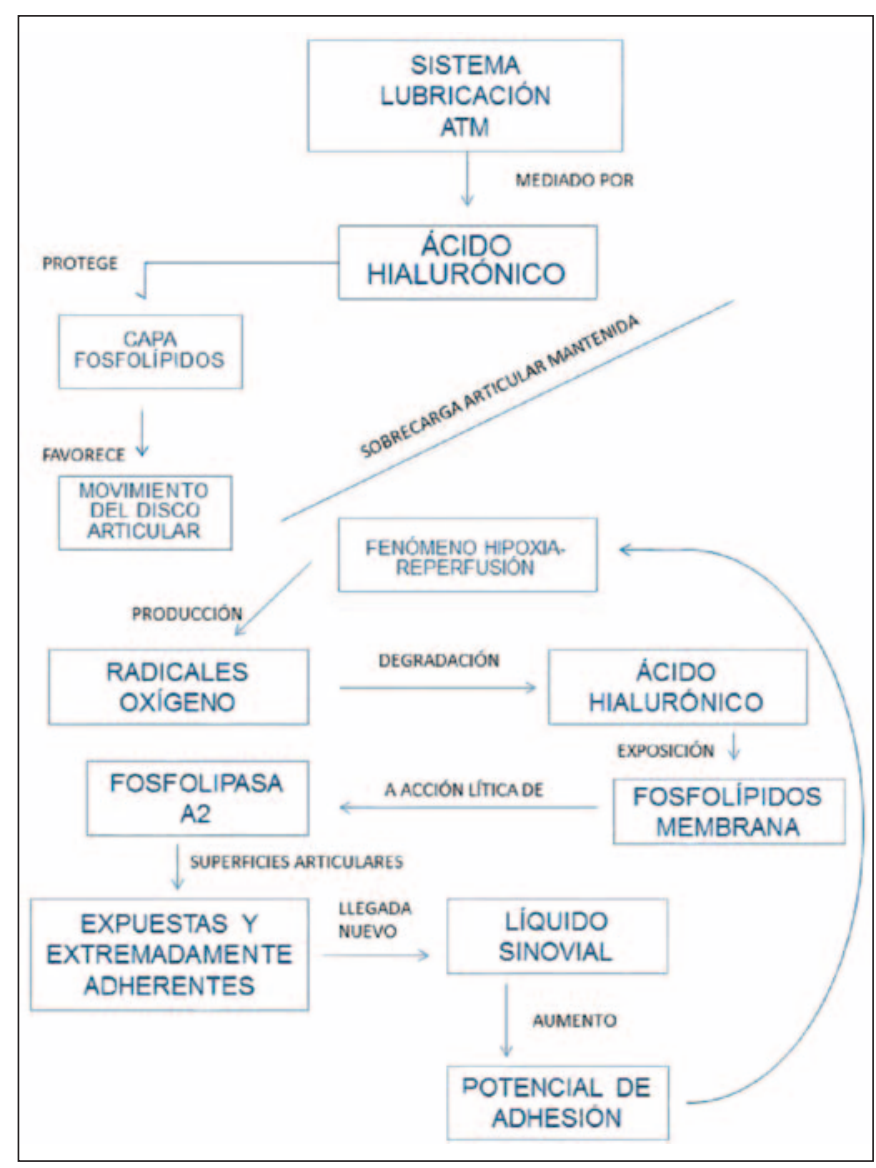

Fig. 1. Descripción del sistema de lubricación de la articulación temporomandibular e hipótesis etiológica de la producción del aumento de la adhesividad del disco articular a las superficies articulares. 
sobrecarga muscular o articular, onicofagia, sostener o mordisquear objetos con la boca y posturas asimétricas (5).

Además la presencia de adherencias discales repetidas y prolongadas puede conducir a la fijación permanente del disco a una de las superficies articulares provocando una adhesión discal. Las adherencias discales presentan una historia característica donde el paciente relata una restricción pasajera de la apertura bucal seguido de un único ruido articular fuerte tipo chasquido o click, recuperando luego la normal movilidad y funcionamiento articular (1) produciéndose la lubricación de la ATM. El episodio se produce generalmente en las mañanas o luego de una carga estática mantenida (1).

En las $\mathrm{AD}$, la fijación es permanente pudiendo ocasionar disminución en el rango de movimiento articular, dependiendo del lugar en que se producen. Cuando las adhesiones discales afectan a una ATM pueden generar una desviación o deflexión hacia el lado homolateral en el movimiento de apertura ocasionado por una hipomovilidad articular del lado afectado (1) pero, cuando afectan a ambas articulaciones, el movimiento puede ser rectilíneo. Los síntomas son fácilmente reproducibles y puede acompañarse o no de dolor, lo cual está asociado principalmente a los intentos por realizar el movimiento articular distendiendo los ligamentos e inflamando la articulación. Al palpar la articulación se pueden manifestar ruidos articulares (4), los cuales van a depender de la posición en que se encuentre el disco adherido.

Las AD pueden provocarse por la unión del disco a la fosa articular o por la unión del disco al cóndilo mandibular, colapsando así el espacio articular superior o el espacio articular inferior, respectivamente.

En el caso de las adhesiones del disco a la fosa articular, el movimiento de apertura se puede encontrar restringido por la limitación en la traslación del cóndilo a través de la superficie anterior de la fosa articular debido a una restricción mecánica (10). Si el disco articular se encuentra adelantado y esta adhesión se mantiene por un periodo largo de tiempo, los ligamentos articulares tienden a distenderse producto del esfuerzo por realizar un movimiento completo produciéndose el paso del cóndilo sobre la superficie posterior del disco ocasionando un ruido tipo click fuerte. En el caso de que el cóndilo además sobrepase la porción anterior del disco articular en el movimiento, se producirá una luxación posterior del disco articular (1), condición conocida como disco fijo. Estos casos son infrecuentes en comparación con una luxación discal anterior pero se encuentran algunos reportes $(11,12)$. Al producirse un estado de disco fijo generalmente no se produce una limitación en la apertura, pero si se pueden encontrar problemas al momento del cierre mandibular, ya que el cóndilo debe sobrepasar la porción anterior o anterior y posterior del disco para lograr el cierre (1). Se ha encontrado un reporte de adhesión discal bilateral con diferentes localizaciones del disco adherido a la fosa articular (7), provocando sintomatología diferente al comparar entre ambas ATM, esto validaría la teoría de que las características clínicas son variables según el lugar de fijación del disco.

Cuando las adhesiones se producen en la cavidad articular inferior su diagnóstico y sospecha clínica son más complicados ya que generalmente no se producen limitaciones en los movimientos, pero pudiera producirse un movimiento de sacudida brusca durante la apertura $(3,13)$ producto de un movimiento rotacional condilar disminuido (1).

La resonancia magnética (RM) es el examen complementario de preferencia para valorar tejidos blandos, tanto en posición estática como dinámica, por lo que es la imagen a elección en la evaluación de una adhesión discal de la ATM $(4,8,9,14)$. Esta técnica entrega información anatómica y funcional y, a la vez evita las radiaciones ionizantes $(8,9)$; en la figura 2 se muestra un ejemplo. La artroscopia es otra técnica útil en la visualización directa de la articulación temporomandibular, lográndose obtener valores diagnósticos muy precisos $(8,9,15)$, pero es una técnica más agresiva.

\section{TRATAMIENTO}

El tratamiento de todas las patologías pertenecientes a los trastornos temporomandibulares tiene como objetivo: una reducción o eliminación del dolor; una 


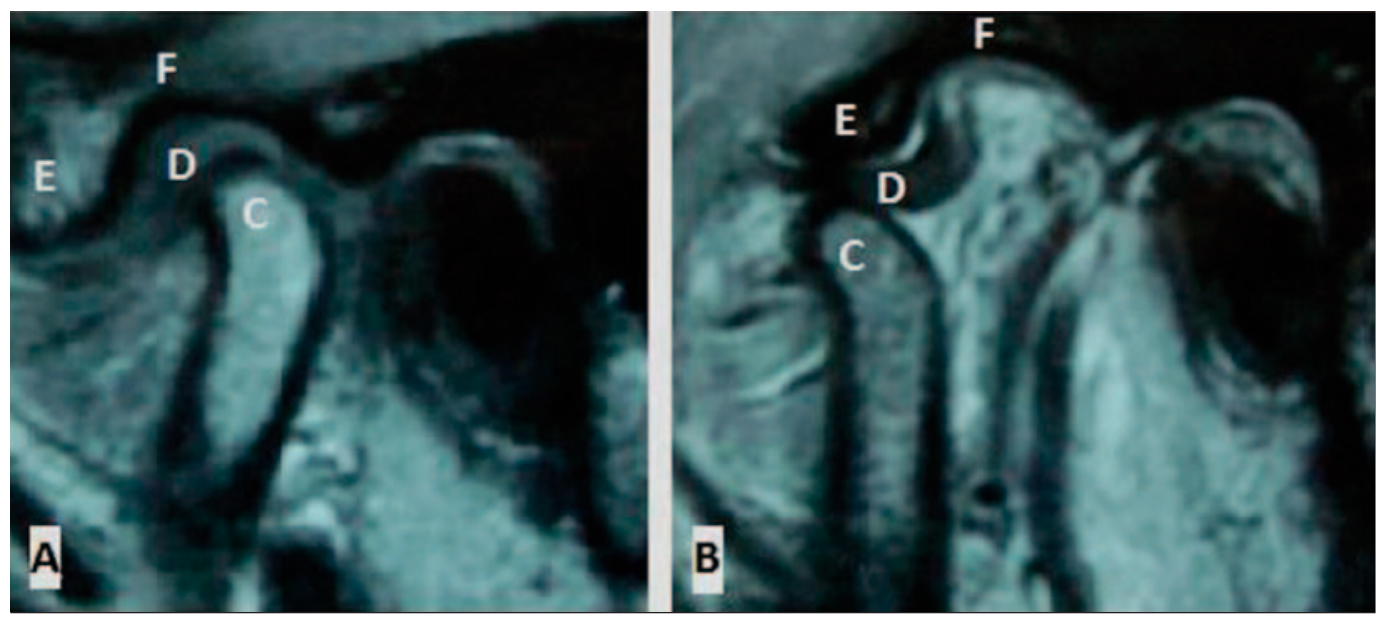

Fig. 2. Resonancia magnética de articulación temporomandibular, en imagen boca abierta el cóndilo mandibular sobrepasa el disco articular evidenciado la adhesión del disco a la fosa mandibular.

A) Imagen boca cerrada;

B) Imagen boca abierta;

C) Cóndilo mandibular;

D) Disco articular;

E) Eminencia articular;

F) Fosa mandibular. restauración de la función mandibular; y una reducción en la necesidad futura de la asistencia médica (4). En el caso de la adhesión discal en la ATM, la literatura propone que los tratamientos son principalmente quirúrgicos siendo la cirugía artroscópica y la artrocentesis las más efectivas $(5,16)$.

La cirugía artroscópica es una técnica mínimamente invasiva donde es posible observar los tejidos directamente y producir la lisis de las fibras adhesivas presentes $(17,18)$. Para poder llevarse a cabo es necesario contar con un artroscopio, una cánula de irrigación y cánulas accesorias para guiar los instrumentos quirúrgicos necesarios $(5,16,17,19)$. El efecto de la cirugía artroscópica puede eliminar la fibrosis e incrementar el rango de apertura bucal mejorando la función mandibular (20).

Por otro lado, la artrocentesis consiste en el lavado de la articulación mediante una técnica de doble punción y el paso de una solución isotónica con una determinada presión $(5,16,17)$, esta presión debe ser la suficiente para dilatar el espacio articular y poder liberar las adhesiones articulares (21). Es una técnica sencilla y al igual que la anterior es mínimamente invasiva $(17,18,22,23)$.

Independiente del tipo de técnica quirúrgica a utilizar, ésta debe ir acompañada de un tratamiento rehabilitador articular posterior, permitiendo así una adaptación de la articulación, una adecuada función y estabilidad posterior (17). Por último, es importante la educación del paciente sobre la patología y las medidas de autocuidado para evitar así una recidiva (4).

\section{BIBLIOGRAFÍA}

1. Okeson JP. Tratamiento de oclusión y afecciones temporomandibulares. $6^{a}$ ed., España: Elsevier; 2008.

2. Flores P, Pena N, Crusoé-Rebello I, Dantas J, Dias $\mathrm{V}$, Macedo J et al. Temporomandibular Joint Disc Adhesion Without Mouth-Opening Limitation. J Oral Maxillofac Surg 2008;66:551-4.

3. Flores P, Farias D, Pena N, Dantas J, Pita de Melo D, Silva C. Adhesión del Disco de la ATM. Reporte de un caso. Acta Odontol Venez 2007;45(supl 4):1-4.

4. Aragón MC, Aragón F, Torres LM. Temporomandibular joint dysfunction. Rev Soc Esp Dolor 2005; 12:429-35.

5. Valmaseda E, Gay C. Diagnóstico y tratamiento de la patología de la articulación temporomandibular. ORL-DIPS 2002;29(supl 2):55-70.

6. Kaminishi RM, Davis CL. Temporomandibular joint arthroscopic observations of superior space adhesions. Oral Maxillofac Surg Clin North Am 1989; 1:103-9. 
7. Campos PSF, Pena N, Nogueira L, Margarida I, Crusoé-Rebello I, Dantas Jet al. Adesão do disco associada a hipoplasia do côndilo da articulação temporomandibular: relato de caso. Rev Imagem 2007;29(supl 2):79-2.

8. Mendoza L, Cañete E, Velilla O. Resonancia magnética de la articulación temporomandibular. Radiología 2008;50:377-85.

9. López J, Chimenos E, Blanco A, Reselló X, Jané E. Diagnóstico por la imagen de los trastornos de la articulación craneomandibular. Av Odontoestomatol 2005;21(supl 2):71-8.

10. Rao VM, Liem MD, Farole A, Razek AA. Elusive "stuck" disk in the temporomandibular joint: diagnosis with MR imaging. Radiology 1993;189: 823-7.

11. Blankestijn J, Boering G. Posterior dislocation of the temporomandibular disc. Int J Oral Surg 1985; $14: 437-43$.

12. Gallagher DM. Posterior dislocation of the temporomandibular joint meniscus: report of three cases. J Am Dent Assoc 1986;113:411-5.

13. Nitzan D, Etsion I. Adhesive force: the underlying cause of the disc anchorage to the fossa and/or eminence in the temporomandibular joint. A new concept. Int J Oral Maxillofac Surg 2002;31:94-9.

14. Venetis G,Pilavaki M, Triantafyllidou K, Papachristodoulou A, Lazaridis N, Palladas P et al. The value of magnetic resonance arthrography of the temporomandibular joint in imaging disc adhesions and perforations. Dentomaxillofacial Radiology 2011;40:84-90.

15. Cabo García R, Grau I, Uribazo A, Pérez O. Generalidades del manejo quirúrgico de los trastornos temporomandibulares con técnicas poco invasivas. Rev Haban cienc méd [online] 2009;8 (supl 4):0-0.

16. Zhang S, Liu X, Yang C, Cai X, Chen M, Haddad $M$ et al. Intra-articular adhesions of the temporomandibular joint: Relation between arthroscopic findings and clinical symptoms. BMC Musculoskelet Disord 2009;10:70.

17. Monje-Gil F, Nitzan D, González-García R. Temporomandibular joint arthrocentesis. Review of the literature. Med Oral Patol Oral Cir Bucal 2012; 17(supl 4):e575-81.

18. Hase M. Adhesions in the temporomandibular joint: Formation and significance. Aust Dent J 2002; 47(supl 2):163-9.

19. Nardini L, Manfredini D, Ferronato G. Arthrocentesis of the temporomandibular joint: a proposal for a single-needle technique. Oral Surg Oral Med Oral Pathol Oral Radiol Endod 2008;106:483-6.

20. Zhang S, Huan D, Liu X, Yang C, Undt G, Haddad $M$ et al. Arthroscopic Treatment for Intra-Articular Adhesions of the Temporomandibular Joint. J Oral Maxillofac Surg 2011;69:2120-7.

21. Yura S, Totsuka Y, Yoshikawa T, Inoue N. Can arthrocentesis release intracapsular adhesions? Arthroscopic findings before and after irrigation under sufficient hydraulic pressure. J Oral Maxillofac Surg 2003;61:1253-6.

22. Vasconcelos B, Bessa-Nogueira R, Studart N. Temporomandibular joint arthrocententesis: evaluation of results and review of the literature. Rev Bras Otorrinolaringol 2006;72(supl 4):634-8.

23. Kaneyama K, Segami N, Shin-Ichi T, Fujimura K, Sato J, Nagao T. Anchored disc phenomenon with a normally positioned disc in the temporomandibular joint: Characteristics and behaviour. Br J Oral Maxillofac Surg 2007;45:279-83.

\section{CORRESPONDENCIA}

Mg. Verónica Iturriaga

Facultad Odontología

Universidad de La Frontera

Manuel Montt, 115

Temuco, Chile

E-mail: veronica.iturriaga@ufrontera.cl 